\title{
Influência de diferentes níveis de salinidade nas características sensoriais do tomate ${ }^{1}$
}

\author{
Waleska M. Eloi ${ }^{2}$, Sergio N . D uarte ${ }^{3}$, Tales M. Soares ${ }^{4}$ \& Énio F. de F. e Silva ${ }^{5}$
}

\section{RESUMO}

O bjetivou-se, com este trabalho, avaliar o efeito de diferentes níveis de salinidade no solo provocados pelo uso da fertirrigação, nas características sensoriais de frutos do tomateiro. 0 experimento foi conduzido em duas etapas, a primeira desenvolvida em laboratório e a segunda realizada em estufa plástica. A primeira etapa consistiu de testes preliminares que possibilitaram a construção de curvas artificiais de salinização, visando à realização do processo de salinização artificial do solo; na segunda etapa estudouse a evolução dos diferentes níveis iniciais de salinidade do solo, implementados por meio da aplicação de fertilizantes, de acordo com a marcha de absorção de nutrientes pela cultura. Os níveis iniciais de salinidade utilizados foram: 1,$5 ; 2,5 ; 3,5 ; 4,5 ; 5,5$ e $6,5 \mathrm{dS} \mathrm{m}^{-1}$. Para a análise sensorial dos frutos realizou-se o teste hedônico com 39 provadores. Verificou-se que os índices de salinidade utilizados não interferiram na aceitação do produto.

Palavras-chave: ambiente protegido, índices de aceitabilidade, Lycopersicum esculentum Mill

\section{Influence of different levels of salinity on sensory characteristics of tomato}

\begin{abstract}
With the objective of evaluating the effect of different salinity levels in the soil provoked by the use of fertigation, on the sensory characteristics of tomato fruits, an experiment was conducted in two stages, being the first developed in laboratory and the second one accomplished in plastic greenhouse. The first stage consisted of preliminary tests that made possible the construction of artificial curves of salinization, seeking accomplishment of the process of artificial salinization of the soil. In the second stage the evolution of the different initial levels of soil salinity was studied, implemented through the application of fertilizers in agreement with the absorption of nutrients for the crop. The levels of salinity used were: 1.5, $2.5,3.5,4.5,5.5$ and $6.5 \mathrm{dS} \mathrm{m}^{-1}$. For the fruit sensory analysis the hedonic test with 39 individuals was performed. It was found that the levels of salinity used did not interfer in the acceptance of the product.
\end{abstract}

Key words: greenhouse; acceptance indices; Lycopersicum esculentum Mill

${ }^{1}$ Parte da Tese de doutoramento do primeiro autor

2 IFCE Campus Sobral, Área de Recursos Naturais, Av. Dr. Guarany 317, Cidao, CEP 62040-730, Sobral, CE. Fone: (88) 3112-8100. E-mail: waleska@ifce.edu.br

${ }^{3}$ DER/ESALQ, Av. Pádua Dias, 11 CP: 9, Piracicaba, SP. Fone: (19) 3447-8543. E-mail: snduarte@esalq.usp.br

${ }^{4}$ CCAAB/U FRB, Rua Rui Barbosa, 710, Centro, CEP. 44380-000, Cruz das Almas, BA, Fone: (75) 36212798 E-mail: talesmiler@gmail.com

${ }^{5}$ DTR/U FRPE, R. Dom Manoel de Medeiros, Dois Irmãos, CEP 52171-900, Recife, PE, Fone: (81) 3320-6261. E-mail: enio.silva@dtr.ufrpe.br 


\section{INTRODUÇÃO}

Dentre as hortaliças produzidas no Brasil, o tomateiro (Lycopersicum esculentum Mill) apresenta-se como a principal, tanto no aspecto econômico quanto no social (Martins et al., 1992). Ocupa uma área superior a 58 mil hectares com produção anual em torno de 3,5 milhões de toneladas (Camargo et al., 2007).

Conforme dados do IBGE (2000), a maioria dos cultivos sob condições protegidas (casa de vegetação), se encontra nos Estados de São Paulo e Minas Gerais. Entre as hortaliças mundialmente cultivadas para consumo in natura e, sobretudo, industrializados, o tomate se sobressai, razão porque é considerado de produção e utilização universal (Camargo et al., 2007).

O cultivo em ambiente protegido é uma das alternativas fundamentais para garantir o aumento da produtividade das olerícolas; entretanto, o manejo inadequado da irrigação, a adição de fertilizantes em altas dosagens e a inexistência de chuvas promotoras de lixiviação, para o excesso de sais aplicados via água de irrigação podem trazer, como consequência, a salinização dos solos neste ambiente, prejudicando a qualidade dos frutos. Apesar das vantagens quando se cultiva em ambiente protegido, potencializa-se o surgimento de um processo muito comum nas regiões áridas e semiáridas: a salinização (Dias et al., 2007).

As dimensões nas quais os produtos alimentícios podem se diferenciar envolvem tanto aspectos intrínsecos de qualidade (perceptíveis pelos sentidos humanos durante o consumo) e relacionados às sensações sensoriais do consumidor (Révillion et al., 2007). Davies \& Maw (1972) citam que a qualidade do tomate condicionada pelo sabor tem sido medida pela proporção adequada entre açúcares redutores (glicose e frutose) e ácidos livres. Segundo Mencarelli \& Saltveit Jr. (1988), frutos de alta qualidade são caracterizados por conter mais do que $0,32 \%$ de acidez total titulável (ATT) e $3 \%$ de sólidos solúveis totais (SST).

Do ponto de vista da ciência dos alimentos, a qualidade é composta pelas características que diferenciam unidades individuais de um produto, sendo significante a determinação do grau de aceitabilidade pelo comprador. O conceito de qualidade do tomate se refere àqueles atributos que o consumidor, consciente ou inconscientemente, estima que o produto deve possuir. Desta forma, são considerados os atributos físicos, sensoriais e a composição centesimal, que devem estar associados para melhor entendimento das transformações que afetam ou não a qualidade do produto (Chitarrra, 1994).

Borguini \& Silva (2005) encontraram diferenças significativas em nível de $5 \%$ de probabilidade nos atributos sabor e aspecto geral quando submeteram amostras de tomate, cv. Débora e Carmen cultivadas nos sistemas convencional e orgânico, à análise sensorial pela escala hedônica. Com relação à avaliação do sabor, a média atribuída ao tomate Carmem produzido organicamente foi menor que aquela atribuída ao tomate cultivado convencionalmente. Em relação ao aspecto geral, a maior nota foi atribuída ao tomate Carmem produzido organicamente, que diferiu significativamente $(\mathrm{p}<0,05)$ apenas do tomate Carmem convencional.
O despertar para a qualidade na agricultura tem sido uma busca incansável nos tempos atuais (Simões et al., 2008), assim a ausência de informações técnico-científicas sobre as características sensoriais do tomate de mesa, cultivado em ambientes com salinidade provocada pelo uso de fertilizantes na água de irrigação, levou à realização da presente pesquisa, com o objetivo de avaliar a influência de possíveis níveis de salinidade utilizados para o cultivo do tomate cv. Débora em relação à aceitabilidade do produto pelo consumidor.

\section{MATERIAL E MÉTODOS}

A metodologia utilizada foi adaptada de Silva et al. (2000), sendo a primeira etapa do experimento desenvolvida no Laboratório de Solos e a segunda realizada em estufa plástica, localizada no Departamento de Engenharia Rural da ESALQ/ USP, situada a $22^{\circ} 42^{\prime}$ 'de latitude sul e $47^{\circ} 38^{\prime}$ de longitude oeste, a uma altitude de $540 \mathrm{~m}$., em Piracicaba, no período entre 20 de novembro de 2006 e 09 de março de 2007.

A primeira etapa consistiu de testes preliminares que possibilitaram a construção de curvas artificiais de salinização visando à realização do processo de salinização artificial do solo.

Para construção das curvas de salinização utilizou-se uma solução nutritiva, sugerida por Furlani et al. (1999). A opção de se utilizar uma solução nutritiva está de acordo com o cultivo fertirrigado, desde que corrigidos previamente os níveis dos nutrientes no solo. Respeitando-se os tipos de sais para macronutrientes e as concentrações originais da solução nutritiva utilizada no estádio produtivo, produziram-se soluções com concentrações salinas crescentes. Em função do nível de condutividade elétrica desejada na solução calculou-se a quantidade de sais a ser adicionada mediante a Eq. 1, proposta por Richards (1954).

$$
\mathrm{C}=640 \mathrm{CE}_{\mathrm{s}}
$$

em que:

$$
\begin{aligned}
& \mathrm{C} \text { - concentração de sais fertilizantes, } \mathrm{mg} \mathrm{L}^{-1} \\
& \mathrm{CE}_{\mathrm{s}} \text { - condutividade elétrica da solução preparada, } \mathrm{dS} \mathrm{m}^{-1}
\end{aligned}
$$

A partir de uma solução padrão com CE teórica de 10,5 dS m-1, com equivalentes $6,72 \mathrm{~g} \mathrm{~L}^{-1}$ de concentração salina, foram tomadas alíquotas para cada nível de salinidade.

Utilizaram-se 21 soluções. A concentração dessas soluções variou de 0 até $6,72 \mathrm{~g} \mathrm{~L}^{-1}$, com intervalos de $0,32 \mathrm{~g} \mathrm{~L}^{-1}$, o que corresponde, respectivamente, às salinidades teóricas variando de 0 até $10,5 \mathrm{dS} \mathrm{m}^{-1}$, com intervalos de $0,5 \mathrm{dS} \mathrm{m}^{-1}$, com base na Eq. (1). A amostra em branco $\left(0 \mathrm{mg} \mathrm{L}^{-1}\right)$ correspondeu à condutividade elétrica inicial da água sem a adição dos fertilizantes. A partir dessas amostras preparadas determinouse a condutividade elétrica real das soluções, utilizando-se um condutivímetro. A curva que relaciona a concentração de fertilizantes e a condutividade elétrica das soluções foi estabelecida por meio de um diagrama de dispersão, onde foram plotados os valores da concentração de sais fertilizantes versus condutividade elétrica encontrada. 
Posteriormente, o solo foi coletado, peneirado e seco ao ar e só então acondicionado em vasos de $20 \mathrm{~L}$, em cuja sua base perfurada havia uma camada de envelope (brita + manta geotextil) de $2 \mathrm{~cm}$. O material de solo utilizado foi de um perfil classificado como Latossolo Vermelho fase arenosa, proveniente do campus da ESALQ e denominado Série "Sertãozinho". Os níveis iniciais de salinidade utilizados foram: $\mathrm{S}_{1}=1,5 \mathrm{dS} \mathrm{m}^{-1} ; \mathrm{S}_{2}=2,5 \mathrm{dS} \mathrm{m}^{-1} ; \mathrm{S}_{3}=3,5 \mathrm{dS} \mathrm{m}^{-1} ; \mathrm{S}_{4}=4,5 \mathrm{dS} \mathrm{m}^{-1}$; $\mathrm{S}_{5}=5,5 \mathrm{dS} \mathrm{m}^{-1}$ e $_{6}=6,5 \mathrm{dS} \mathrm{m}^{-1}$.

A umidade do solo foi elevada até a máxima capacidade de retenção e, concomitantemente, a ele foram adicionados os sais diluídos na água com o objetivo de se obter seis níveis de condutividade elétrica do extrato de saturação, com base na curva de salinização, construída na primeira etapa; três dias após a adição das diversas soluções salinas, amostras de solo foram retiradas dos vasos nas camadas de 0 a 0,15 m e de 0,15 a $0,30 \mathrm{~m}$, após uma raspagem na qual se eliminaram $2 \mathrm{~cm}$ da superfície do solo, pois, de acordo com Sonneveld (1988), a salinidade se mede principalmente na zona abaixo da superfície; na superfície se teriam valores altíssimos, mesmo nos tratamentos de menor salinidade, em que a planta não responde a esses picos de salinidade e, sim, à salinidade dos pontos nos quais existe água mais facilmente disponível. Determinou-se, a partir dessa amostragem, a condutividade elétrica do extrato de saturação real, ou seja, aquela que corresponde aos valores encontrados no solo.

$\mathrm{Na}$ segunda etapa do experimento os tratamentos, ministrados na cultura do tomateiro, foram compostos da combinação de dois fatores: salinidade inicial do solo, com seis níveis e dois tipos de manejo de fertirrigação $\left(M_{1}-\right.$ de acordo com a marcha de absorção da cultura e $\mathrm{M}_{2}$ - com controle da condutividade elétrica da solução do solo). Os tratamentos propostos foram dispostos em 48 parcelas e o delineamento estatístico adotado foi o de blocos aleatorizados completos com quatro repetições, ficando os fatores estudados arranjados no esquema fatorial $6 \times 2$.

Utilizou-se a cultivar Débora Plus, tipo longa vida, com hábito de crescimento indeterminado e início de colheita por volta dos 105 dias após a semeadura e ciclo de 150 dias; o espaçamento utilizado foi de 0,50 m entre plantas e 1,20 m entre fileiras. As plantas foram conduzidas com apenas um ramo principal, eliminando-se as ramificações laterais, tão logo formadas. A operação de desbrota foi realizada duas vezes por semana e se realizou a poda apical ao redor de $1,80 \mathrm{~m}$ de altura. A temperatura média do ar no interior da casa de vegetação foi de $21,0{ }^{\circ} \mathrm{C}$, encontrado-se as mínimas entre 9 e $19^{\circ} \mathrm{C}$ e as máximas variaram de 20 a $38^{\circ} \mathrm{C}$; a umidade relativa média foi de $53,3 \%$.

Adotou-se um sistema de irrigação por gotejamento utilizando-se emissores do tipo autocompensante, com vazão nominal de $4 \mathrm{~L} \mathrm{~h}^{-1}$, os quais foram previamente avaliados em campo sob condições normais de operação. O sistema de irrigação apresentou coeficiente de uniformidade de distribuição de $94,13 \%$. Realizou-se o manejo de irrigação com base em dados de potencial mátrico da água no solo, obtidos de tensiômetros instalados a $0,15 \mathrm{~m}$ de profundidade, e da curva característica de retenção de água no solo.

A aplicação da irrigação foi realizada de acordo com a recomendação de Alvarenga (2004), sempre que a tensão se encontrava entre 15 a $20 \mathrm{kPa}$. A quantidade de água aplicada era suficiente para elevar a umidade à capacidade de campo, calculada mediante as médias das leituras tensiométricas, para cada tratamento.

A aplicação dos fertilizantes foi feita via água de irrigação, sendo os tratamentos diferenciados em função dos manejos $M_{1}$ e $M_{2}$. O manejo $M_{1}$ baseou-se na marcha de absorção da cultura apresentada por Alvarenga (2004), o qual recomenda os seguintes valores totais para adubação de 1.000 plantas de tomateiro, via fertirrigação: $\mathrm{N}=35 \mathrm{~kg}, \mathrm{P}=14 \mathrm{~kg}, \mathrm{~K}=75 \mathrm{~kg}$ e Ca $=10 \mathrm{~kg}$. A frequência da fertirrigação foi semanal, de acordo com o sugerido pelo citado autor.

Para os tratamentos referentes ao manejo $\mathrm{M}_{2}$, realizou-se a fertirrigação semanalmente, a fim de manter os níveis iniciais de condutividade elétrica na solução do solo, para cada tratamento. Calculou-se a quantidade de fertilizantes aplicada para que a solução do solo se mantivesse no nível de CE inicial $\left(1,5 ; 2,5 ; 3,5 ; 4,5 ; 5,5\right.$ ou $\left.6,5 \mathrm{dS} \mathrm{m}^{-1}\right)$. Para este cálculo, utilizouse a fórmula de mistura de soluções (Eq. 2); desta forma, a condutividade elétrica na solução do solo foi o controlador do manejo da fertirrigação. Quando não foi necessário aplicar fertilizantes, realizou-se apenas a irrigação.

$$
\mathrm{CE}_{\mathrm{c}}=\frac{\mathrm{V}_{\mathrm{cc}} \mathrm{CE}_{\mathrm{esd}}-\mathrm{V}_{\mathrm{a}} \mathrm{CE}_{\mathrm{a}}}{\mathrm{V}_{\mathrm{r}}}
$$

em que:

$\mathrm{V}_{\mathrm{cc}}$ - Volume de água armazenada no solo, à capacidade de campo, $\mathrm{L}$

$\mathrm{CE}_{\text {esd }}$ - Condutividade elétrica do extrato de saturação do solo desejada, $\mathrm{dS} \mathrm{m} \mathrm{m}^{-1}$

$\mathrm{V}_{\mathrm{a}}$ - Volume atual de água armazenada no solo, $\mathrm{dS} \mathrm{m}^{-1}$

$\mathrm{CE}_{\mathrm{a}}$ - Condutividade elétrica atual do solo, $\mathrm{dS} \mathrm{m} \mathrm{m}^{-1}$

$\mathrm{V}_{\mathrm{r}}{ }^{\mathrm{a}}$ - Volume de água no solo necessário para se atingir a capacidade de campo,L

$\mathrm{CE}_{\mathrm{c}}$-Condutividade elétrica da solução de correção para se atingir a $\mathrm{CE}_{\text {esd }}, \mathrm{dS} \mathrm{m}^{-1}$

Segundo com o programado para o manejo $M_{1}$ ou com o necessário para o manejo $\mathrm{M}_{2}$, os fertilizantes utilizados (nitrato de cálcio, nitrato de potássio, fosfato monoamônico, fosfato monopotássico, sulfato de potássio e sulfato de magnésio) foram diluídos nas águas de irrigação armazenadas em caixas de PVC, correspondentes a cada tipo de manejo.

A condutividade elétrica da solução foi medida após o término de cada evento de irrigação utilizando-se, para extração da solução, cápsulas porosas sob vácuo. Os valores de condutividade elétrica, determinados mediante a solução extraída pelas cápsulas porosas, foram corrigidos para a umidade de saturação, conforme sugerido por Silva et al. (2000) na Eq. 3.

$$
\mathrm{C}_{\text {estimada }} \mathrm{cp}=\frac{\mathrm{CcpUcp}}{\mathrm{Us}}
$$

em que:

$\mathrm{C}_{\text {estimada }} \mathrm{cp}$ - condutividade elétrica ou concentração de íons no extrato de saturação, estimada a partir dos valores medidos 
na solução do solo obtida com extrator de cápsula, $\mathrm{dS} \mathrm{m} \mathrm{m}^{-1} \mathrm{ou}$

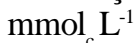

Ccp - condutividade elétrica ou concentração de íons na solução do solo, obtida com extrator com cápsula porosa, $\mathrm{dS} \mathrm{m}$ ${ }^{1}$ ou mmol $\mathrm{L}^{-1}$

Ucp - umidade do solo no momento da retirada da solução com o extrator com cápsula porosa, $\mathrm{g} \mathrm{g}^{-1}$

Us - umidade do solo na pasta saturada, $\mathrm{g} \mathrm{g}^{-1}$

Realizou-se a análise sensorial dos tomates a partir do teste hedônico (Meilgaard et al., 1999), mediante uma ficha de avaliação contendo 7 itens: 1.desgostei muito, 2.desgostei moderadamente, 3.desgostei ligeiramente, 4. não gostei nem desgostei, 5.gostei ligeiramente, 6.gostei moderadamente, 7.gostei muito, com observação da qual gostou muito e menos, e porque. Todos os tratamentos (6 6 2) junto com uma testemunha (frutos adquiridos no mercado local) foram avaliados, em número de 4 amostras por provador, segundo o delineamento em blocos incompletos Tipo II, com $\mathrm{t}=13, \mathrm{k}=3$, $\mathrm{r}=6, \mathrm{~b}=26, \lambda=1 \mathrm{eE}=0,72$.
Solicitou-se que as notas 1 e 7 correspondessem a: desgostei muito (nota 1); gostei muito (nota 7) e que se descrevessem quais os atributos que caracterizavam essas notas.

Os tomates foram lavados manualmente em água corrente e, em seguida, colocados em bacias de $10 \mathrm{~L}$ com 20 ppm de hipoclorito de sódio em pó, específico para alimentos, durante 10 min para a sanitização; depois, foram enxaguados em água corrente para a retirada do excesso de hipoclorito, evitando possíveis sabores residuais interferentes na análise sensorial e drenados para a retirada do excesso de água.

As amostras de tomate foram servidas em pratinhos descartáveis de polipropileno, aleatorizados com números de 3 dígitos; cada provador recebeu 4 amostras, sendo utilizados os 13 tratamentos já descritos.

Os tomates foram avaliados por um grupo de 39 consumidores, dos quais 26 do sexo feminino e 13 do sexo masculino; a divisão por sexo foi aleatória. Os resultados impetrados foram submetidos à análise de variância e teste de Tukey.

Tabela 1. Teste de diferença do controle em tomates

\begin{tabular}{|c|c|c|c|c|c|c|c|c|c|c|c|c|c|}
\hline \multirow{2}{*}{ Provador } & \multicolumn{13}{|c|}{ Tratamentos } \\
\hline & $\mathrm{T} 1$ & $\mathrm{~T} 2$ & T3 & T4 & T5 & T6 & $\mathrm{T7}$ & T8 & T9 & $\mathrm{T} 10$ & $\mathrm{~T} 11$ & $\mathrm{~T} 12$ & $\mathrm{~T} 13$ \\
\hline 1 & 5 & 6 & & 4 & & & & & & 6 & & & \\
\hline 2 & & 7 & 4 & & 3 & & & & & & 5 & & \\
\hline 3 & & & 6 & 3 & & 7 & & & & & & 6 & \\
\hline 4 & & & & 6 & 7 & & 5 & & & & & & 5 \\
\hline 5 & 3 & & & & 7 & 3 & & 6 & & & & & \\
\hline 6 & & 5 & & & & 7 & 6 & & 7 & & & & \\
\hline 7 & & & 7 & & & & 6 & 2 & & 3 & & & \\
\hline 8 & & & & 1 & & & & 7 & 3 & & 3 & & \\
\hline 9 & & & & & 7 & & & & 2 & 6 & & 6 & \\
\hline 10 & & & & & & 5 & & & & 6 & 6 & & 6 \\
\hline 11 & 6 & & & & & & 2 & & & & 7 & 6 & \\
\hline 12 & & 7 & & & & & & 1 & & & & 6 & 1 \\
\hline 13 & 4 & & 5 & & & & & & 5 & & & & 3 \\
\hline 14 & 5 & 6 & & 6 & & & & & & 6 & & & \\
\hline 15 & & 4 & 3 & & 5 & & & & & & 5 & & \\
\hline 16 & & & 7 & 7 & & 6 & & & & & & 6 & \\
\hline 17 & & & & 6 & 6 & & 7 & & & & & & 6 \\
\hline 18 & 7 & & & & 5 & 3 & & 6 & & & & & \\
\hline 19 & & 4 & & & & 4 & 3 & & 1 & & & & \\
\hline 20 & & & 4 & & & & 7 & 7 & & 3 & & & \\
\hline 21 & & & & 7 & & & & 6 & 5 & & 4 & & \\
\hline 22 & & & & & 3 & & & & 6 & 3 & & 6 & \\
\hline 23 & & & & & & 5 & & & & 6 & 7 & & 5 \\
\hline 24 & 6 & & & & & & 5 & & & & 6 & 7 & \\
\hline 25 & & 4 & & & & & & 3 & & & & 5 & 6 \\
\hline 26 & 7 & & 7 & & & & & & 7 & & & & 7 \\
\hline 27 & 5 & 6 & & 7 & & & & & & 7 & & & \\
\hline 28 & & 5 & 7 & & 6 & & & & & & 3 & & \\
\hline 29 & & & 6 & 3 & & 7 & & & & & & 7 & \\
\hline 30 & 2 & & & 4 & 5 & & 5 & & & & & & 4 \\
\hline 31 & 6 & & & & 7 & 6 & & 7 & & & & & \\
\hline 32 & & 6 & & & & 3 & 1 & & 2 & & & & \\
\hline 33 & & & 6 & & & & 4 & 7 & & 5 & & & \\
\hline 34 & & & & 5 & & & & 4 & 6 & & 3 & & \\
\hline 35 & & & & & 5 & & & & 6 & 7 & & 6 & \\
\hline 36 & & & & & & 7 & & & & 6 & 7 & & 6 \\
\hline 37 & 7 & & & & & & 7 & & & & 7 & 7 & \\
\hline 38 & & 7 & & & & & & 7 & & & & 6 & 6 \\
\hline 39 & 7 & . & 7 & & & & & & 6 & & & & 5 \\
\hline
\end{tabular}




\section{RESULTADOS E DISCUSSÃO}

Os dados obtidos no teste se encontram na Tabela 1; pelos resultados obtidos, constata-se que não houve diferença estatística entre os tratamentos (Tabela 2).

Tabela 2. Resultados do teste hedonico em tomates submetidos a diferentes tratamentos

\begin{tabular}{|c|c|}
\hline Tratamentos* & Médias \\
\hline $\mathrm{T} 1$ & $5,38 \mathrm{a}$ \\
\hline $\mathrm{T} 2$ & $5,46 \mathrm{a}$ \\
\hline T3 & $5,77 \mathrm{a}$ \\
\hline T4 & $4,92 \mathrm{a}$ \\
\hline T5 & $5,23 \mathrm{a}$ \\
\hline T6 & $5,23 \mathrm{a}$ \\
\hline $\mathrm{T7}$ & $4,92 \mathrm{a}$ \\
\hline T8 & $5,38 \mathrm{a}$ \\
\hline T9 & $4,69 a$ \\
\hline T10 & $5,15 a$ \\
\hline T11 & $5,23 \mathrm{a}$ \\
\hline T12 & $6,15 a$ \\
\hline T13 & $5,07 \mathrm{a}$ \\
\hline \multicolumn{2}{|c|}{ 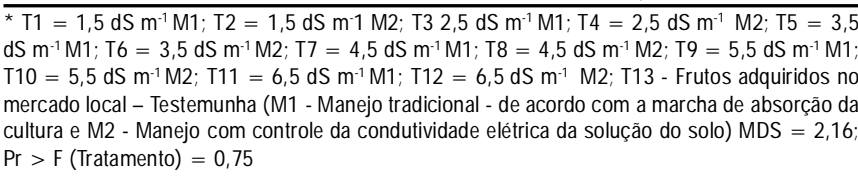 } \\
\hline
\end{tabular}

A Tabela 3 apresenta as porcentagens de notas e os índices de aceitabilidade para os diferentes tratamentos; notas acima de 4 são consideradas de aceitabilidade da amostra; pode-se observar que todos os tratamentos obtiveram altos índices de aceitabilidade, com o tratamento T12 atingindo o maior índice, $100 \%$ de aceitabilidade, seguido dos T1, T3, T5 e T13, e o tratamento T4, o menor índice, ou seja, $62 \%$; ressalta-se que não houve diferença estatística nos resultados.

Os atributos descritos pelos provadores que melhor definiram essas diferenças entre os índices de aceitabilidade foram: sabor suave, saboroso, perfeito balanceamento entre a acidez e a doçura, textura e sabor agradável, macio, suculento, doce, firme, vermelho mais intenso, menos ácido, levemente salgado, carnudo para os tratamentos que receberam a maior porcentagem de aceitabilidade, tratamentos T12, T1, T3, T5 e T13; e muito maduro, pouco consistente, macento, cor pálida, casca dura, sabor estranho, muito mole, seco, esfarela na boca, ácido, sabor desagradável, passado, muita fibra e verde, para o tratamento $\mathrm{T} 4$, o qual recebeu a menor porcentagem de aceitabilidade. Fato interessante a ser observado pelos provadores foi que eles descreveram a amostra menos ácida como a mais doce (Figura 1), razão do atributo doce em relação à amostra T12.

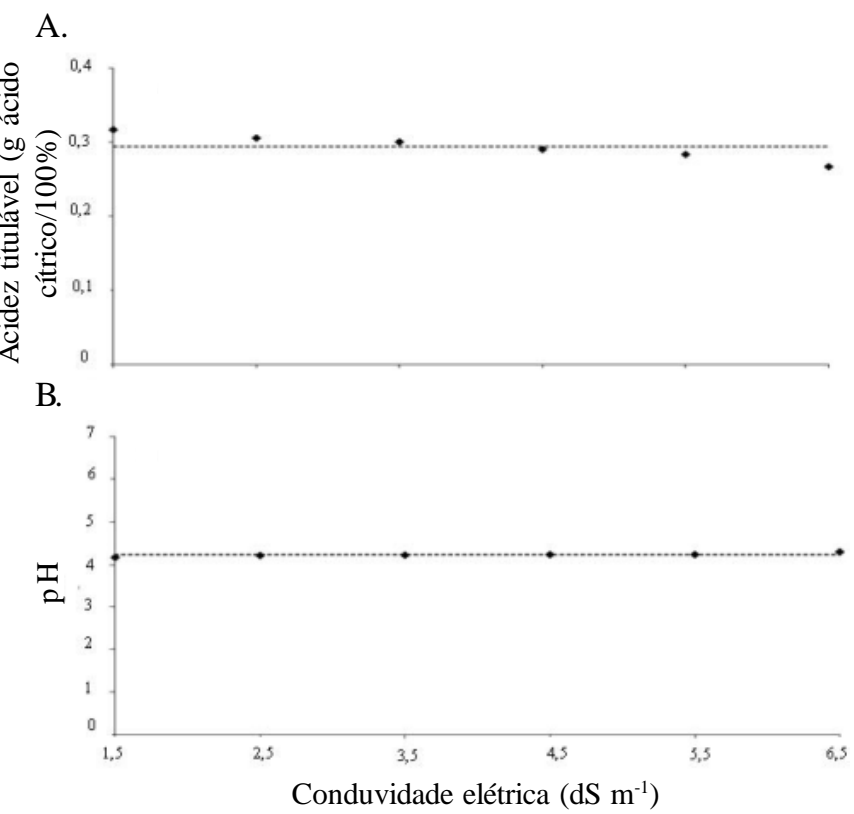

Figura 1. Valores médios de acidez titulável (A) e pH (B) para os diferentes níveis de condutividade elétrica

A nota média global da escala hedônica foi de 5,27 e o menor índice de aceitabilidade calculado de acordo com Teixeira et al. (1987) foi de 62\%. Segundo Anzaldúa-Morales (1994), os índices de aceitabilidade acima de $60 \%$ são considerados adequados.

\section{ConClusÃo}

1. Para a análise sensorial realizada, concluiu-se que os índices de salinidade utilizados não interferiram na aceitação do tomate pelo consumidor.

\section{AgRADECIMENTO}

Ao CNPq, por ter financiado a pesquisa.

Tabela 3. Porcentagens de notas e índices de aceitabilidade dos diferentes tratamentos

\begin{tabular}{cccccccccccccc}
\hline Notas & T1 & T2 & T3 & T4 & T5 & T6 & T7 & T8 & T9 & T10 & T11 & T12 & T13 \\
1 & - & - & - & 8 & - & - & 8 & 8 & 8 & - & - & - \\
2 & 8 & - & - & - & 8 & - & 8 & 8 & 15 & - & - & - \\
3 & 8 & - & 8 & 15 & 15 & 23 & 8 & 8 & 8 & 31 & 23 & - & 8 \\
4 & 8 & 31 & 15 & 15 & - & 8 & 8 & 8 & - & - & 8 & - & 8 \\
5 & 23 & 15 & 8 & 15 & 31 & 23 & 23 & - & 23 & 8 & 23 & 8 & 23 \\
6 & 23 & 31 & 31 & 23 & 15 & 15 & 23 & 23 & 31 & 46 & 15 & 69 & 46 \\
7 & 31 & 23 & 38 & 23 & 31 & 31 & 23 & 46 & 15 & 15 & 31 & 23 \\
$* *$ & 77 & 69 & 77 & 62 & 77 & 69 & 69 & 69 & 69 & 69 & 69 & 100 & 77 \\
\hline
\end{tabular}

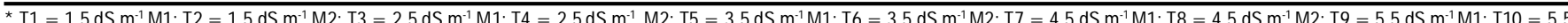
$\mathrm{dS} \mathrm{m}^{-1} \mathrm{M} 2 ; \mathrm{T} 11=6,5 \mathrm{dS} \mathrm{m} \mathrm{m}^{-1} \mathrm{M} 1 ; \mathrm{T} 12=6,5 \mathrm{dS} \mathrm{m}^{-1} \mathrm{M} 2 ; \mathrm{T} 13$ - Frutos adquiridos no mercado local - Testemunha (M1 - Manejo tradicional - de acordo com a marcha de absorção da cultura e M2 - Manejo com controle da condutividade elétrica da solução do solo)

** Soma de porcentagens de provadores para as notas de 5 a 7, consideradas índices de aceitabilidade para os diferentes tratamentos 


\section{LITERATURA CITADA}

Alvarenga, M. A. R. Tomate: Produção em campo, em casa de vegetação e em hidroponia. Lavras: UFLA, 2004. 400p.

Anzaldúa-Morales, A. La evaluación sensorial de los alimentos en la teoria y la práctica. Zaragoza: Acribia, 1994. 198p.

Borguini, R. G.; Silva, M. V. Características físico-químicas e sensoriais do tomate (lycopersicon esculentum) produzido por cultivo orgânico em comparação ao convencional. Alimentos e Nutrição Araraquara, v.16, p.355-361, 2005.

Camargo, G. A.; Haj-Isa, N.; Queiroz, M. R. de. Avaliação da qualidade de tomate seco em conserva. Revista Brasileira de Engenharia Agrícola e Ambiental, v.11, p.521-526, 2007.

Chitarra, M. I. F. Colheita e qualidade pós-colheita de frutos. Informe Agropecuário, Belo Horizonte, v.17, p. 8-18, 1994.

Davies, J. N.; Maw, G. A. Metabolism of citric and malic acids during ripening of tomato fruit. Journal of Science Food Agriculture, v.23, p.969-976, 1972.

Dias, N. da S.; Duarte, S. N.; Teles Filho, J. F.; Yoshinaga, R. T. Salinização do solo por aplicação de fertilizantes em ambiente protegido. Irriga, v.12, p.135-143, 2007.

Furlani, P. R.; Silveira, L. C. P.; Bolonhezi, D.; Faquin, V. Cultivo hidropônico de plantas. Campinas: Instituto Agronômico, 1999. 52p. Boletim Técnico 180

IBGE - Instituto Brasileiro de Geografia e Estatística. Levantamento sistemático da produção agrícola: pesquisa mensal de previsão e acompanhamento das safras agrícolas no ano civil. 2000. <http://www.ibge.gov.br/>. 12 Jun. 2006.
Martins, G.; Castellane, P. D.; Volpe, C. A.; Banzatto, C. A. Uso da casa de vegetação com cobertura plástica na tomaticultura de verão. Desenvolvimento e produção. Horticultura Brasileira, v.10, p.60-64, 1992.

Meilgaard, M.; Civille, B.; Carr, T. Sensory evaluation techniques. 3.ed. Boca Raton: CRC Press, 1999. 416p.

Mencarelli, F.; Saltveit, Jr. M.E. Ripening of mature-green tomato fruit slices. Journal of American Society for Horticultural Science, v.113, p.742-745, 1988.

Révillion, J. P. P; Flôres,S. H.; Wilk, E. de O.; Badejo, M. S.; Mercali, G. D.; Gandolfi, L. M.; Alles, M. J. L.; Mariot, R. F.; Campos, S. U. de.; Alberti, S. S.; Romero, A. M. Qualidade sensorial de vinhos tintos finos do Rio Grande do Sul comparados aos importados da Argentina e Chile. Ciência e Tecnologia de Alimentos, v.27, p.177-180, 2007.

Richards, L. A. Diagnosis and improvement of saline and alkali soils. Washington: United States Salinity Laboratory, 1954. 160p.. Agriculture Handbook, 60

Silva, E. F.F.; Anti, G. R.; Carmello, Q. A. de C.; Duarte, S. N. Extratores de cápsulas porosas para o monitoramento da condutividade elétrica e do teor de potássio na solução de um solo. Scientia Agrícola, v.57, p.785-789, 2000.

Simões, R. de O.; Faroni, L. R. A.; Queiroz, D. M. de. qualidade dos grãos de café (Coffea arabica L.) em coco processados por via seca. Caatinga, v.21, p.139-146, 2008.

Sonneveld, C. The salt tolerance of greenhouse crops. Neth. Journal of Agricultural Science. v.36, p.63-67, 1988.

Teixeira, E; Meineri, E. M.; Barbeta, P. O. A. Análise sensorial de alimentos. Florianópolis: UFSC, 1987. 180p. 